\title{
Étude écophysiologique des parasitoses à Cymothoadiens
}

\author{
par B. ROMESTAND \\ Laboratoire de Physiologie des Invertébrés, \\ Université des Sciences et Techniques du Languedoc, F. 34060 Montpellier Cedex.
}

\section{Résumé.}

Une étude écophysiologique de certaines parasitoses à Cymothoadiens a permis de mettre en évidence :

- l'existence de glandes latéro-œsophagiennes produisant un principe anticoagulant actif sur le sang des poissons hôtes, l'absorption et la métabolisation des dérivés de l'hémoglobine (hématine et fer) étant dévolue à l'hépatopancréas ;

- l'existence de réactions immunologiques entre les poissons (sains et parasités) et les Cymothoadiens ;

- l'existence des variations biochimiques, histologiques, hématologiques et biométriques chez les poissons parasités.

\section{Summary.}

Ecophysiological study on the parasitic action of Cymothoids.

An ecophysiological study on certain parasitisms by Cymothoids allows the elucidation of the following points:

- occurence of oesophagien-side glands producing an anticoagulant effect on the blood of host, the absorption and metabolism of haemoglobin derivatives (hematin and iron) are performed by the hepatopancreas;

- existence of immunological reactions between host and Cymothoids;

- existence of biochemical, histological, haematological and biometrical variations among fishes infected by these parasites.

Accepté le 27 février 1979. 


\section{Introduction}

Dans le règne animal ou végétal, il existe des espèces parasites qui durant toute ou partie de leur existence, se nourrissent aux dépens d'autres êtres vivants.

Les Cymothoidae (Crustacés, Isopodes, Flabellifères) appartiennent à cette catégorie d'individus ; ce sont des ectoparasites de poissons marins ou d'eaux douces appelés parfois vulgairement \& poux de poissons ; ils sont caractérisés par un hermaphrodisme protérandrique et sont parasites à l'état adulte.

Depuis une dizaine d'années, à la suite du travail de Trilles (1968), nous possédions une riche information sur la biologie des Cymothoidae du golfe du Lion; en outre, de nombreux problèmes liés au parasitisme Cymothoadiens s'y voyaient posés ; cependant, bien des recherches restaient à poursuivre dans ce domaine.

Les études (1) que nous avons entreprises relèvent toutes du domaine de l'écophysiologie parasitaire, de l'écologie et de la parasitologie marine; elles ont eu deux orientations principales, ainsi :

- les unes ont été consacrées aux problèmes nutritionnels (hématophagie) soulevés par cette famille de parasites;

- les autres ont concerné certains problèmes fondamentaux relatifs aux intéractions hôte/parasite; nous avons par exemple essayé de préciser si (et de quelle façon) la présence des Cymothoadiens induit l'existence de réactions immunologiques spécifiques, des variations d'ordres biochimique, histologique, hématologique et biométrique des hôtes?

\section{Matériel et méthodes}

Les Cymothoidae sont des Crustacés qui appartiennent à l'ordre des Isopodes et au sous-ordre des Flabellifères. Après une courte période de vie libre qui fait suite à la mise bas, les larves (pulli) se déplacent activement dans l'eau à la recherche des poissons hôtes, sur lesquels elles vont se fixer. Sur ces derniers, où elles accomplissent leur croissance et leur développement, chaque espèce occupe des positions caractéristiques et bien déterminées. Les Cymothoidae du golfe du Lion peuvent être ainsi répartis en trois catégories écologiques (Trilles, 1968): Cymothoadiens buccaux, de surface et branchiaux $(P l . I ; 1$ à 8$)$. savoir :

Nous n'avons étudié que des représentants des deux premières catégories à

- Cymothoadiens buccaux: Emetha audouini (Edwards, 1840); Meinertia parallela (Otto, 1828); Meinertia oestroides (Risso, 1826).

(1) Cet article est le résumé d'une thèse de Doctorat d'Etat, mention Biologie Animale, soutenue le 22 septembre 1978 devant l'Université des Sciences et Techniques du Languedoc. 

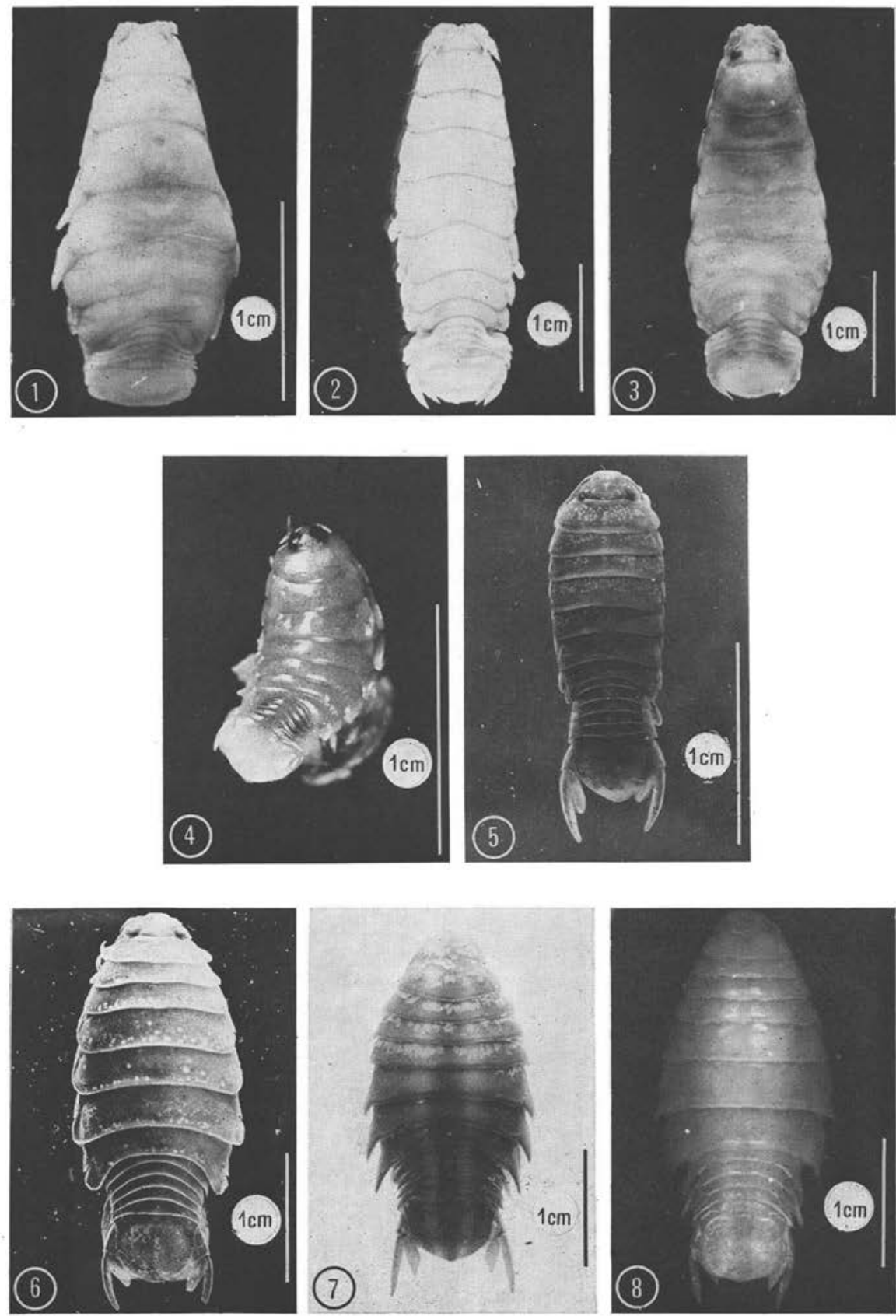

Planche I. Quelques Isopodes Cymothoidae du Golfe du Lion.

1: Emetha audouini femelle; 2: Meinertia parallela femelle; 3: Meinertia oestroides femelle; 4 : Mothocya epimerica femelle ; 5 : Anilocra physodes mâle; 6 : Anilocra physodes femelle; 7 : Nerocila orbignyi femelle; 8 : Nerocila maculata femelle. 
- Cymothoadiens de surface : Anilocra physodes (L., 1758); Nerocila orbignyi (Guerin-Meneville, 1829).

Les poissons sur lesquels nous avons récolté ces différents parasites, appartiennent au super-ordre des Téléostéens et à diverses familles parmi lesquelles celles des $(P l . I I ; 9$ à 11) : 1758).

- Sparidae: Boops boops (Linnaeus, 1758); Pagellus erythrinus (Linnaeus,

- Maenidae: Maena maena (Linnaeus, 1758); Maena smaris (Linnaeus, 1758).

- Mugilidae: Chelon labrosus (Risso, 1826); Liza (Liza) aurata (Risso, 1826).

Pour ce travail, nous avons également retenu d'autres Téléostéens que nous avons qualifié de poissons non hôtes potentiels de Cymothoadiens. Il s'agit d'espèces sur lesquelles on ne rencontre normalement pas de Cymothoidae, tout au moins en Méditerranée occidentale (indépendamment du parasitisme occasionnel), accidentel ou de capture), par exemple :

- Congridae : Conger conger [(Artedi, 1758) (Linnaeus, 1758)].

- Carangidae: Trachurus mediterraneus (Steindachner, 1868).

Tous ces poissons et les parasites ont été pêchés au large des ports d'Agde et de Sète (Hérault).

Meinertia oestroides et surtout Meinertia parallela sont spécifiques de Boops boops, Emetha audouini de Maena maena ou de Maena smaris, Nerocila orbignyi des poissons appartenant à la famille des Mugilidae. Anilocra physodes a une répartition ubiquiste; on peut la rencontrer sur Boops boops, Maena smaris et Pagellus erythrinus.

Le sang des poissons a été prélevé sur les lieux de récolte par ponction intracardiaque, à l'aide de pipettes Pasteur stériles.

L'hémolymphe des Cymothoadiens a été ponctionnée dans le vaisseau dorsal. Dans les deux cas, après la prise en masse du caillot, les tubes sont centrifugés, les sérums décantés additionnés de merthiolate à $1 \%$ et conservés en tubes stériles à $-20^{\circ} \mathrm{C}$.

- Pour la recherche du facteur anticoagulant et pour préciser son mécanisme d'action, nous avons réalisé un certain nombre de tests de coagulation plasmatique, à l'aide de fiches Stago (2) (Romestand et Trilles, 1976).

- Les doubles diffusions ont été conduites selon la méthode de Ouchterlony (1948).

- Pour le dosage des protéines, nous avons utilisé le réactif de Folin-Ciocalteu (Lowry et al., 1951). La détection d'activités enzymatiques a été réalisée par le procédé Api Zym (3).

(2) Laboratoire Stago, 92600 Asnières.

(3) Société Api, 69621 Villeurbanne. 

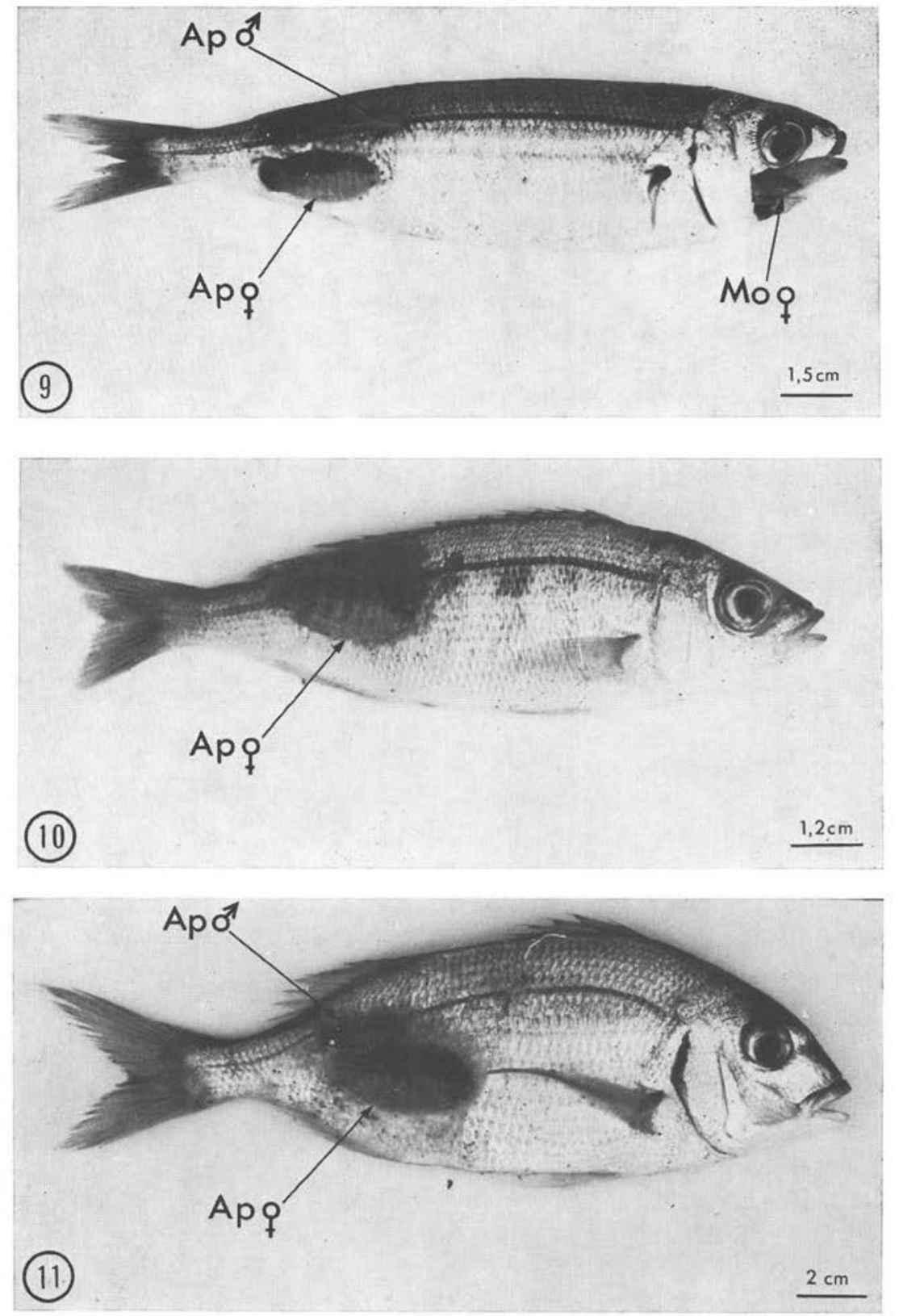

Planche II. Quelques poissons hôtes potentiels des Isopodes Cymothoidae. 9: Boops boops parasitée simultanément par Meinertia oestroides (femelle) et par Anilocra physodes (mâle et femelle). 10: Maena maena parasitée par Anilocra physodes (femelle). 11: Pagellus erythrinus parasité par Anilocra physodes (mâle et feməlle). Ap $=$ Anilocra physodes $;$ Mo $=$ Meinertia oestroides. 
- Les glucides du sérum et du foie des poissons ont été extraits après action de l'éthanol et du chloroforme (Johnston et Davies, 1972) et dosés par la méthode à l'orcinol sulfurique (Montreuil et Spick, 1963).

- Pour le dosage des lipides sériques, nous avons utilisé le réactif à la phosphovanilline (coffret «Merkotest» Merck). Les dosages des lipides du foie des poissons ont été réalisés par la méthode pondérale de Folch et al. (1957).

- L'ion chlore $(\mathrm{Cl}-)$ a été dosé par «l'aminco Cotlove Titrator» dont le principe est basé sur le temps que mettent les ions chlore (contenus dans la solution) à se combiner avec les ions argent produits par l'oxydation électrochimique d'une électrode génératrice.

Les ions sodium $(\mathrm{Na}+)$, potassium $(\mathrm{K}+)$ et calcium $(\mathrm{Ca}++)$ ont été dosés au photomètre à flamme de type Eppendorf.

L'ion magnésium $(\mathrm{Mg}++)$ a été analysé par spectrophotométrie d'absorption atomique sur Pelkin Elmer, modèle 303.

- Les techniques histologiques employées ont été des plus courantes :

Fixation : dans le liquide de Halmi ou dans le liquide de Bouin alcoolique (Martoja R. et Martoja M., 1967).

Colorations : nous avons surtout employé l'hémalun éosine, l'hémalun picro indigo carmin, la coloration trichromique de Masson (variante de Goldner) et le trichrome de Mallory (Martoja R. et Martoja M., 1967).

- Pour la mise en évidence des produits résultant de la dégradation du sang des poissons au niveau de l'appareil digestif des Cymothoadiens, nous avons utilisé des techniques histochimiques moins usuelles et notamment:

- la caractérisation de l'hémoglobine par la benzidine, après transformation en méthémoglobine (Gabe, 1968);

- la détection du fer $(\mathrm{Fe}+++)$ sels ferriques (sexquioxydes) par la méthode au bleu de Prusse (Gabe, 1968).

la détection simultanée du fer ferrique $(\mathrm{Fe}+++)$ et du fer ferreux $(\mathrm{Fe}++$, protoxyde) par la méthode au bleu de Turnbull (Gabe, 1968).

- Sur les poissons, outre les tests d'hémostases, nous avons réalisé les analyses hématologiques suivantes :

- numération des érythrocytes et des leucocytes par comptage électronique au Coulter Counter (modèle D), après dilution du sang dans la solution de Hendrick's (Hesser, 1960). Des frottis sanguins ont également été réalisés et colorés selon la méthode de Giemsa/Pappenheim (Gabe, 1968); 1958) ;

- dosage de l'hémoglobine par la méthode à la cyanméthémoglobine (Hainline,

- détermination de l'hématocrite en tubes capillaires héparinés (microhématocrite). 
Les différentes valeurs de l'hématocrite, du dosage de l'hémoglobine et de la numération des érythrocytes, nous ont permis de calculer la concentration corpusculaire moyenne en hémoglobine $(\mathrm{CCMH})$, l'hémoglobine corpusculaire moyenne (HCM) et la valeur globulaire moyenne (VGM).

- L'étude de la relation taille-poids et de la croissance des poissons sains et parasités, nous a conduit à mesurer leur taille et leur poids. Nous avons également évalué leur âge à partir de l'étude des otolithes selon la technique de Christensen (1964).

Pour l'analyse des résultats (et notamment pour leur comparaison), différentes méthodes statistiques ont été utilisées parmi lesquelles:

- les tests de Student pour la comparaison des moyennes (Martin, 1967);

- les formules de Le Cren (1951) pour l'étude de la relation taille-poids, avec les tests de Mayrat $(1959,1967,1970)$, pour la comparaison des droites d'allométrie ;

- les analyses de la covariance selon la méthode de Lellouch et Lazar (1974) pour la comparaison des courbes de croissance.

\section{L'hématophagie des Cymothoadiens}

D'après un certain nombre d'observations et en particulier celles de Schioedte (1868), Brian (1912), De Scalzi (1941), Legrand (1952), Bowman (1960), Trilles (1968) et Monod 1976), il semble que l'on puisse affirmer que le régime alimentaire des Cymothoadiens est un régime presque exclusivement hématophagique.

La bouche est située en position ventrale et entourée par des pièces buccales puissantes (pour dilacérer les téguments) et acérées (pour percer les vaisseaux sanguins nourriciers); le tube digestif quant à lui, présente $(P l . I I I ; 12)$ :

- un œsophage court, revêtu d'un épithélium stratifié et muni de fibres musculaires radiaires réglant la contraction et la dilatation de l'organe ;

- un estomac complexe pourvu de plaques triturantes et de saillies chitinisées;

- un intestin rectiligne aboutissant à l'anus ;

- six caecums hépatopancréatiques débouchent à la limite estomac-intestin ;

- deux diverticules intestinaux postérieurs.

Mais les Cymothoadiens, pour pouvoir se nourrir convenablement et utiliser au mieux les différents composants du sang des poissons, disposent surtout (comme cela existe chez certains Insectes hématophages) d'une substance capable d'empêcher ou de retarder la coagulation sanguine. L'existence d'un tel facteur et le lieu éventuel de sa sécrétion, ont été précisés à partir de l'expérience suivante (Romestand et Trilles, 1976) : 

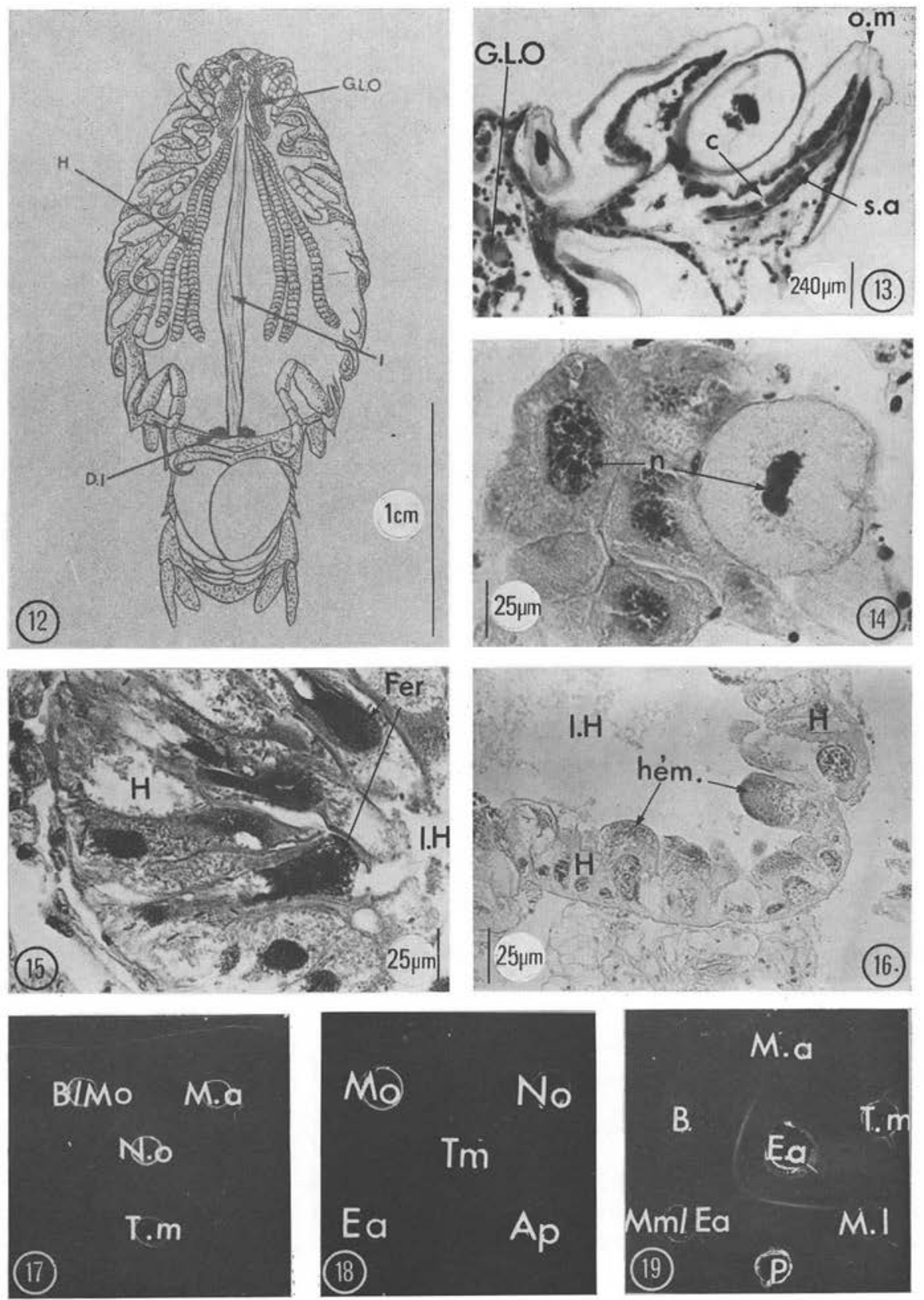
des extraits salins de céphalon (in toto), d'hépatopancréas, d'intestin et de diverticules intestinaux postérieurs ont été mis au contact de sang de poissons hôtes potentiels de Cymothoidae, de poissons non hôtes potentiels et même de sang humain.

Dans ces conditions, on peut remarquer que les extraits d'hépatopancréas, d'intestin et de diverticules intestinaux postérieurs n'ont pas de pouvoir anticoagulant; par contre les extraits de céphalon manifestent une forte activité anticoagulante sur le sang total des poissons hôtes, des poissons non hôtes potentiels et même sur le sang humain.

Comme l'ont successivement montré Montalenti (1949), Siniscalco (1951), Geitler (1953), Gabe (1960) et Fain-Maurel (1966), le céphalon des Cymothoadiens est le siège d'une accumulation de cellules glandulaires exocrines, généralement groupées en rosettes; elles se répartissent en trois groupes distincts respectivement:

- rostral,

- latéro-ganglionnaire,

- latéro-œsophagien.

Ce dernier, selon Gabe (1960), jouerait un rôle dans la prise de nourriture. Effectivement, des expériences d'hémostase à partir d'extraits de glandes latéro-œsophagiennes ont donné des résultats identiques à ceux obtenus à partir du céphalon in toto.

En définitive, l'action anticoagulante mise en évidence au niveau du céphalon semble par conséquent surtout attribuable à la présence de ces glandes. Elles sont paires, situées de part et d'autre de l'œsophage $(P l . I I I ; 12)$ et constituées par de grosses cellules de $200 \mu \mathrm{m}$ présentant une activité sécrétoire cyclique. Parmi ces dernières, certaines sont transparentes à noyau bien visible, d'autres ont au contraire un cytoplasme rendu opalescent par l'accumulation de nombreux grains de sécrétion (Pl. III ; 14). Des canaux excréteurs multicellulaires (1 par glande) débouchent de part et d'autre de la bouche au niveau des mandibules $(P l . I I I ; 13)$.

\section{Planche III}

12: Représentation schématique du tube digestif de Anilocra physodes. 13: Coupe transversale de la mandibule de Anilocra physodes. 14: Coupe transversale d'une glande latéroœsophagienne de Anilocra physodes. (Deux des trois types cellulaires rencontrés). 15: Détection histochimique du fer (ferrique et ferreux) dans les cellules hépatopancréatiques de Anilocra physodes. 16: Détection histochimique de l'hématine dans les cellules hépatopancréatiques de Anilocra physodes.

$\mathrm{c}=$ canal ; D.I. $=$ diverticules intestinaux; G.L.O. $=$ glandes latéro-œesophagiennes ; $\mathrm{H}=$ hépatopancréas; hém $=$ hématine ; $\mathrm{I}=$ intestin ; $\mathrm{L} . \mathrm{H}=$ lumière hépatopancréatique ; $\mathrm{n}=$ noyau $;$ o.m. $=$ orifice mandibulaire $;$ s.a. $=$ substance anticoagulante.

17, 18, 19: Doubles diffusions en gélose sellon la méthode de Ouchterlony.

A.p. = sérum de Anilocra physodes; $\mathrm{B}=$ sérum de Boops boops; $\mathrm{B} / \mathrm{M} . \mathrm{o}=$ sérum $\mathrm{de}$ Boops boops parasitée par Meinerta oestroides; E. $\mathrm{a}=$ sérum de Emetha audouini; $\mathrm{M} . \mathrm{a}=$ sérum de Mugil auratus; $\mathrm{M} .1=$ sérum de Morone labrax; M.m/E.a = sérum de Maena maena parasitée par Emetha audouini; M.o = sérum de Meinertia oestroides; N.o $=$ sérum de Nerocila orbignyi $; \mathrm{P}=$ sérum de Pagellus erythrinus $; \mathrm{T} . \mathrm{m}=$ sérum de Trachurus mediterraneus. 
On pourrait raisonnablement s'interroger sur le mécanisme d'action exact d'un tel principe anticoagulant. Sommes-nous en présence d'un anticoagulant de type salin qui complexe les ions calcium du sang, ou au contraire d'un facteur agissant sur un ou plusieurs facteurs plasmatiques (extrinsèque ou intrinsèque) de la coagulation ? Pour essayer de répondre à cette question, nous avons envisagé l'étude d'un certain nombre de tests d'hémostase parmi lesquels; temps de : Howell, de Quick, de Céphaline-kaolin, de Stypven, de Stypven-céphaline. Ceci nous a permis de démontrer, dans un premier temps, d'une manière nette, que la substance anticoagulante agit sur les facteurs plasmatiques communs aux deux voies de la coagulation.

Mais pour mieux préciser l'action de ce facteur, d'autres tests ont été nécessaires : temps de thrombine et de thrombine coagulase. La non augmentation de ce dernier en présence d'extraits de glandes latéro-œsophagiennes, permet de déduire que la substance agit sur la thrombine ou sa genèse; elle peut donc être homologuée à une antithrombine de type héparine ou hirudine (Romestand et Trilles, 1976). Nos résultats sont ainsi à rapprocher de ceux de Markwardt et Walsmann (1958) qui mettent en évidence une activité antithrombique de l'hirudine.

Quelle est la nature chimique de cette substance dont l'activité mime celle de l'héparine (mucopolyoside acide à glucosamine) ? Se rapproche-t-elle de l'héparine ? Il semble que ce soit le cas puisque les cellules granuleuses des glandes latéro-œsophagiennes présentent une intense activité A.P.S. positive et le spectre enzymatique des extraits de ces glandes une prédominance d'activités enzymatiques de type glucidase, avec par exemple, une augmentation de l'intensité de la $\beta$-glucuronidase, de l' $\alpha$-glucosidase, de la $\beta$-glucosaminidase, de l' $\alpha$-mannosidase et de l' $\alpha$-glucosidase (tableau $I$ ).

Le sang des poissons hôtes est donc maintenu fluide par action de cette substance anticoagulante, mais comment est-il dégradé et par quel (s) organe (s) est-il absorbé ?

Les processus d'alimentation des Cymothoidae ne sont pas continus mais cycliques (Trilles, 1968 et observations personnelles); ils sont caractérisés par une alternance de processus de succion de sang et d'absorption au niveau des caecums hépatopancréatiques.

Des expériences de digestion du sang in vitro montrent d'ailleurs que l'hépatopancréas (à un fort degré), l'intestin et les diverticules intestinaux postérieurs ( à un plus faible degré) produisent des «ferments hémolytiques » actifs sur les érythrocytes du sang des hôtes.

Les caractérisations histochimiques des produits (hématéine, hématine et fer) résultant de la dégradation de l'hémoglobine sur des individus en état de réplétion (en phase active de digestion) se sont révélées positives au niveau de l'hépatopancréas (Pl. III ; 15 et 16$)$; par contre elles ont été négatives au niveau de l'intestin et des diverticules intestinaux postérieurs.

Le sang ingéré, maintenu fluide grâce à l'intervention du principe anticoagulant des glandes latéro-œsophagiennes, est refoulé par le pharynx et l'œsophage dans l'intestin et les caecums hépatopancréatiques. C'est dans ces derniers que s'effectue la majeure partie de la dégradation de l'hémoglobine en hématine et l'absorption. 
Tableau I. Activités enzymatiques des extraits des glandes latéro-œesophagiennes (G.L.O.) et de l'hépatopancréas chez l'Isopode Cymothoidae Meinertia oestroides (femelle).

\begin{tabular}{|c|c|c|c|c|c|c|c|c|c|c|c|c|c|c|c|c|c|c|}
\hline & 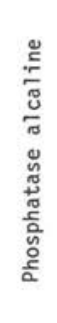 & 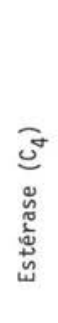 &  & 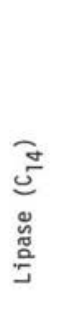 & 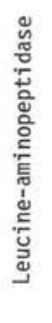 & 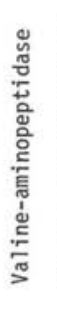 & 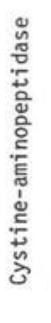 & $\frac{\stackrel{\Xi}{n}}{\stackrel{n}{2}}$ & 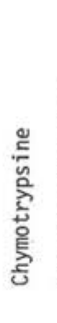 & 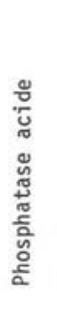 & 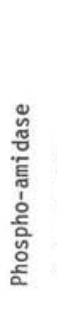 & 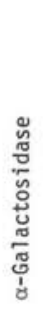 & 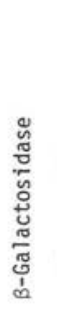 & 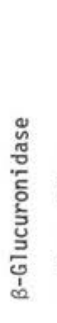 & 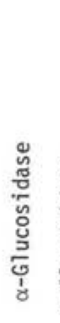 & 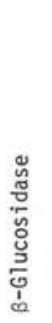 & 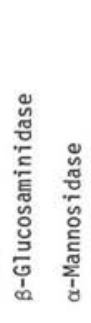 & 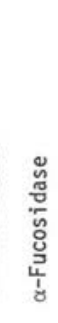 \\
\hline G.L.O, & 5 & 2 & $2^{+}$ & 0 & 4 & 1 & 1 & 0 & $1^{+}$ & 5 & $4^{+}$ & 1 & 0 & $4^{+}$ & 5 & 1 & 52 & 5 \\
\hline HEPATOPANCREAS & 2 & 2 & $2^{+}$ & 0 & 4 & 2 & 1 & 0 & $1^{-}$ & 4 & 5 & 0 & 0 & 1 & 2 & 0 & 21 & 2 \\
\hline
\end{tabular}

Importance quantitative des activités enzymatiques :

-4 à 5 : activité forte.

-2 à 3 : activité moyenne.

- 1: activité faible.

- 0: activité nulle.

L'intestin et les diverticules intestinaux postérieurs préparent la digestion du sang en contribuant à l'hémolyse des érythrocytes. Au niveau de l'hépatopancréas, le groupement prosthétique pigmentaire est certainement décomposé en une substance voisine de l'hémochromogène et en fer. De la décomposition des dérivés de l'hémoglobine et de la digestion intracellulaire des substances protéiques des érythrocytes, il résulte vraisemblablement des lipides, tandis que le fer non métabolisé se dépose dans les cellules hépatopancréatiques.

\section{Les rapports hôtes/parasites}

La plupart des parasites animaux ou végétaux modifient d'une façon plus ou moins nette les organismes des hôtes sur lesquels ils vivent. Il arrive même parfois qu'entre le parasite et son hôte s'établissent des influences réciproques qui peuvent être de diverses natures: immunologiques, biochimiques, histologiques. hématologiques, etc.

Nous avons essayé de les étudier dans le cas des parasitoses à Cymothoidae.

\section{Rapports immunologiques.}

Les poissons présentent une réponse immunitaire comparabe par bien des aspects à celle des Mammifères. Ils sont capables de synthétiser des anticorps contre une grande variété d'antigènes. Des immunoglobulines ont ainsi été signalées par plusieurs 
auteurs, notamment lors des infestations parasitaires chez la truite arc-en-ciel (Hodgins et al., 1967), le saumon (Alexander et al., 1970) et le cyprin (Harris, 1972).

De telles réactions immunologiques existent-elles dans le cas des parasitoses à Cymothoidae? Pour essayer de répondre à cette question nous avons réalisé des doubles diffusions en gélose selon la méthode de Ouchterlony (1948); trois types de réactions ont été envisagées : $(P l . I I I ; 17,18$ et 19):

- réactions directes: elles opposent les protéines sériques des Cymothoadiens à celles de leurs hôtes spécifiques (exemple: Meinertia oestroides/Boops boops) ;

-- réactions indirectes : elles opposent les protéines sériques des Cymothoadiens à celles des poissons hôtes non spécifiques (exemple: Emetha audouini/Boops boops) ;

- réactions témoins: elles opposent les protéines sériques des Cymothoadiens à celles de certains poissons non hôtes potentiels (par exemple: Trachurus mediterraneus ou Morone labrax).

Lors des réactions directes et indirectes et exception faite de celles mettant en jeu les protéines sériques de Mugil auratus [Liza (Liza) auratus], Chelon labrosus et Mugil capito, nous avons toujours obtenu un arc de précipitation. Lors des réactions témoins, nous n'avons par contre jamais observé d'arc de précipitation.

Pour préciser le sens des réactions immunologiques ainsi mises en évidence, nous avons effectué des réactions d'agglutination passive (test au latex) qui ont apporté la preuve qu'elles se produisent toujours chez le poisson (anticorps) et à l'égard du Cymothoadien (antigène). On peut d'ailleurs ajouter :

- qu'elles ne semblent pas entièrement spécifiques puisqu'en effet, elles se manifestent (identité immunologique totale) quel que soit le parasite (buccal, branchial ou de surface) et le poisson considéré, dans la mesure où il ne s'agit pas d'un poisson appartenant à la famille des Mugilidae ou d'un poisson non hôte potentiel de Cymothoidae.

— qu'elles ne semblent se développer qu'à l'égard des Cymothoadiens.

L'absence de réaction immunologique chez les Muges pourrait expliquer que Nerocila orbignyi diffère fondamentalement des autres espèces par le nombre souvent important ( 6 à 8 et même parfois plus) d'individus fixés sur le même poisson ; chez les autres Cymothoadiens, le parasitisme par couple d'individus est en effet normalement la règle. On peut dès lors se demander si l'hyperparasitisme des Mugilidae ne serait pas justement attribuable à l'absence de réactions immunologiques. Ces dernières, quand elles existent, permettraient de limiter le nombre de parasites fixés sur un même poisson, contrôlant ainsi la réalisation d'un certain état d'équilibre entres les Cymothoadiens et leurs hôtes.

\section{Rapports biochimiques.}

Si la littérature concernant les actions parasitaires des Protozoaires et des Helminthes sur les constituants biochmiques des hôtes (Vertébrés supérieurs) est riche 
[cf. en particulier Van Brand (1973)], il n'en est pas de même dans le cas des autres Invertébrés marins parasites de poissons. Dans ce domaine, les travaux consacrés aux Cymothoadiens sont d'ailleurs inexistants.

Nous avons analysé les variations des principaux constituants organiques et minéraux (sériques et hépatiques) des poissons lors des parasitoses à Cymothoadiens (tableau II).

- Constituants organiques: au niveau sérique comme au niveau hépatique, nous avons observé dans tous les cas :

- aucune variation de la teneur en protéines et en oses totaux (différences non significatives) ;

- une diminution de la lipémie et de la teneur en lipides hépatiques.

- Constituants minérauX: au niveau sérique, on observe toujours:

- aucune variation significative de la natrémie et de la chlorémie ;

- une tendance (différences non significatives) à l'hypokaliémie et à l'hypocalcémie ;

— une hypomagnésémie (différences significatives).

En définitive, les Cymothoadiens buccaux ou de surface induisent sur les poissons hôtes :

- une diminution de la teneur en lipides sérique et hépatique;

- une hypomagnésémie.

Or, le magnésium est généralement considéré comme un soporifique (narcotique) aussi bien chez les Vertébrés que chez les Invertébrés [(Robertson, 1954); (Lagarrigue, 1963)] ; on peut donc se demander si l'hypomagnésémie qui caractérise les hôtes parasités ne serait pas à l'origine d'un certain état d'excitation des poissons, qui se traduirait par une augmentation de leur activté natatoire.Toutefois, nos observations sur le comportement des hôtes parasités sont encore trop rares pour nous permettre d'infirmer ou de confirmer cette hypothèse.

\section{INFLUENCES HISTOLOGIQUES :}

Suivant leur localisation sur les poissons, les Cymothoadiens exercent des actions plus ou moins importantes et variées. Nous nous limiterons cependant ici au cas des parasites buccaux: Meinertia oestroides et Emetha audouini.

- Meinertia oestroides (au moins les individus femelles) sont fixées sur le «plancher» lingual, plus précisément au niveau de la langue.

Lors des parasitoses correspondantes, on observe une atrophie progressive de la langue du poisson; elle devient d'abord de plus en plus filliforme, puis très nettement plus courte et trapue $(P l . I V ; 20)$. Cette atrophie peut atteindre $50 \%$ de la longueur de l'appendice initial. 
Parmi les modifications histologiques concomittantes, on peut relever au niveau de l'implantation des pièces buccales $(P l . I V ; 21)$.

- une disparition de l'épiderme (assise génératrice et membrane basale);

- une désorganisation des différentes couches du derme (où les différentes couches ne sont plus visibles;

- une forte réaction hémorragique, avec présence de très nombreux lymphocytes, érythrocytes ;

- une apparition de très nombreuses cavités dans la substance fondamentale de l'os, avec ça et là des cellules plurinucléées $(P l . I V ; 22)$ qui ressemblent aux ostéoclastes.

La régression de la langue de Boops boops parasitées par Meinertia oestroides est très certainement une conséquence de l'hématophagie des parasites (Romestand et Trilles, 1977).

- Emetha audouini est un parasite spécifique des poissons appartenant à la famille des Maenidae. Les individus femelles sont appliqués sur le «plafond buccal » (voûte palatine). Ils induisent des modifications crâniennes ( $P l . V: 23)$;

- élargissement du vomer,

— diminution très nette de l'angle formé par le parasphénoïde et le vomer.

- présence constante de très nombreuses dents vomériennes, groupées en amas sur une sorte de mamelon.

Parmi les modifications histologiques concomittantes, on peut relever :

- au niveau de l'implantation des parasites:

- une disparition plus ou moins importante de l'épiderme ;

- une désorganisation des différentes couches du derme;

— une forte réaction hémorragique.

— en avant de la zone de fixation parasitaire :

- une apparition de dents vomériennes; elles sont relativement fortes et solidement fixées sur l'os vomérien par leur base $(P l . I V ; 24)$.

De nombreuses observations histologiques des voûtes palatines chez des Maenidae non parasités ont été réalisées, afin d'essayer de déceler la présence éventuelle de bourgeons dentaires pré-existants; toutes se sont révélées négatives. On peut donc admettre que ces formations dentaires correspondent certainement à des néoformations vraies.

Les déformations crâniennes et la présence de dents vomériennes peuvent être interprétées comme des caractères adaptatifs en rapport avec une certaine protection des parasites contre le courant buccal des hôtes. 
INFLUENCES HÉMATOLOGIQUES.

Le sang d'un poisson correspond à la plus grande partie de son milieu intérieur et possède des caractéristiques fixes et bien déterminées. Mais de profondes variations peuvent être liées au sexe (Preston, 1960), à la reproduction (Poston, 1966), à l'alimentation (Johansson-Sjöbeck et al., 1967) et au parasitisme (Man, 1952), (Kabata, 1958).

Nous avons donc envisagé l'étude des modifications hématologiques éventuelles en rapport avec le parasitisme Cymothoadien. Nous avons pu noter dans tous les cas (tableau III) :

- une diminution du nombre d'érythrocytes circulants (différences significatives),

- une diminution de l'hémoglobine et de l'hématocrite,

— une stabilité du volume globulaire moyen, de la concentration corpusculaire moyenne en hémoglobine et de l'hémoglobine corpusculaire moyenne,

- une stabilité du nombre des leucocytes circulants.

Si le nombre global des leucocytes reste stable, n'existe-il pas des variations des formules leucocytaires correspondantes? Pour essayer de répondre à cette question, étant donné la grande confusion qui règne dans les descriptions des leucocytes du sang des poissons (Jakowska, 1956), nous avons été amené à établir au préalable notre propre nomenclature, puis les formules leucocytaires correspondantes. Parmi les cellules rencontrées dans le sang circulant on note la présence: - de lymphocytes, - d'éléments mononucléés - d'hémoblastes, - de cellules à noyau polymorphe, - de cellules à granulations éosinophiles et de thrombocytes $\left(^{*}\right)$.

En fonction du parasitisme, on peut mettre en évidente (tableau IV):

- une augmentation (significative) du nombre d'hémoblastes circulants,

- une tendance à l'augmentation des éléments mononucléés,

- une tendance à la diminution du nombre de lymphocytes,

— une stabilisation des cellules à noyau polymorphe et de thrombocytes.

Chez les poissons Téléostéens, on sait que la rate produit des érythrocytes et des globules blancs (Catton, 1951; Yoffey, 1929). Des études pondérales et histologiques de cet organe en fonction du parasitisme par les Cymothoadiens ont mis en évidence :

- une augmentation pondérals de la rate. Elle est de l'ordre de $50 \%$ pour Boops boops et Maena maena; elle est toutefois beaucoup plus faible chez Pagellus erythrinus (de l'ordre de 20 à $25 \%$ ) (tableau IV).

- une hypervascularisation de cet organe, avec apparition de nombreuses néoformations vasculaires $(\mathrm{Pl} . \mathrm{IV} ; 25$ et 26$)$.

(*) Cette catégorie cellulaire a été évaluée hors pourcentage. 


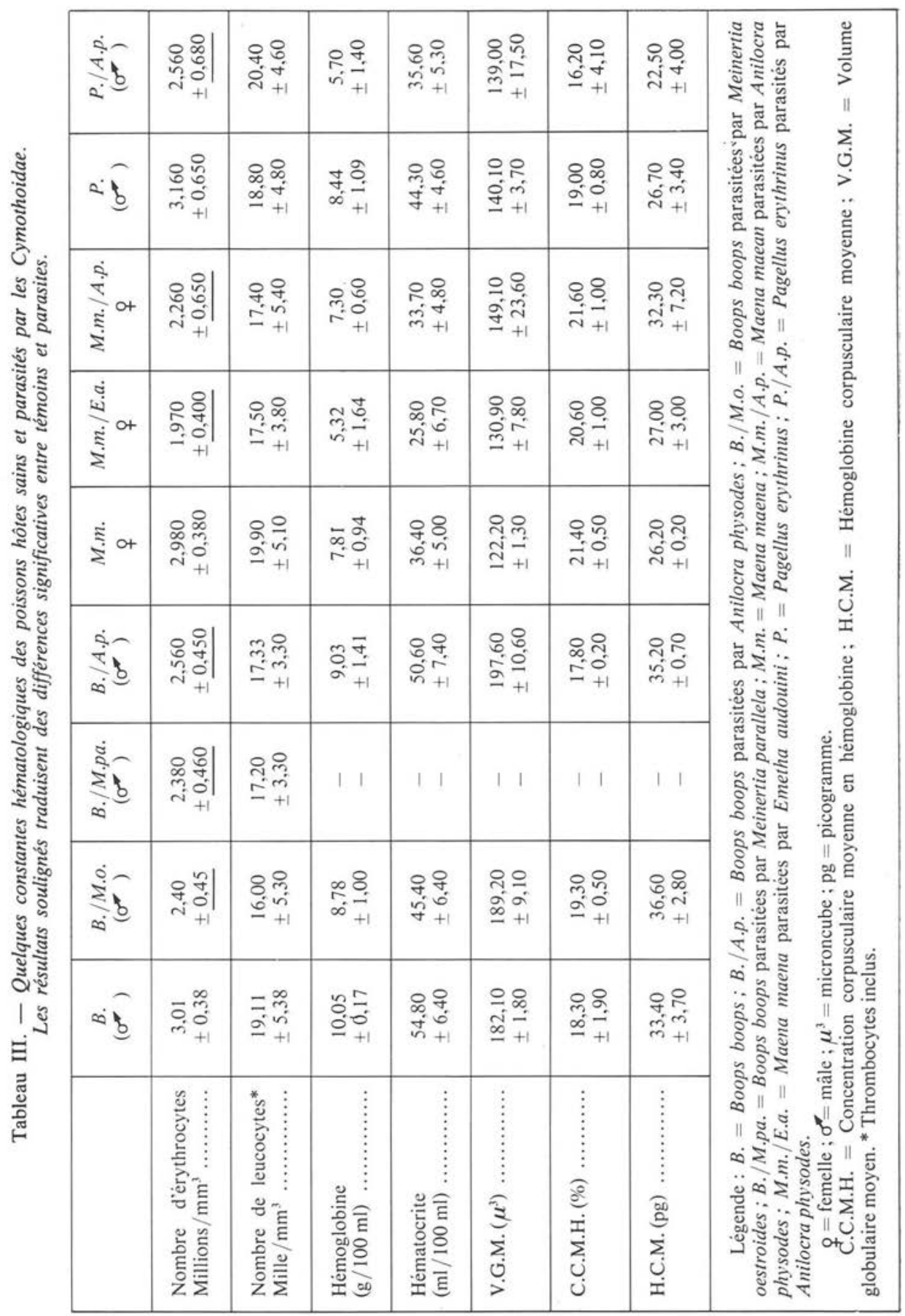




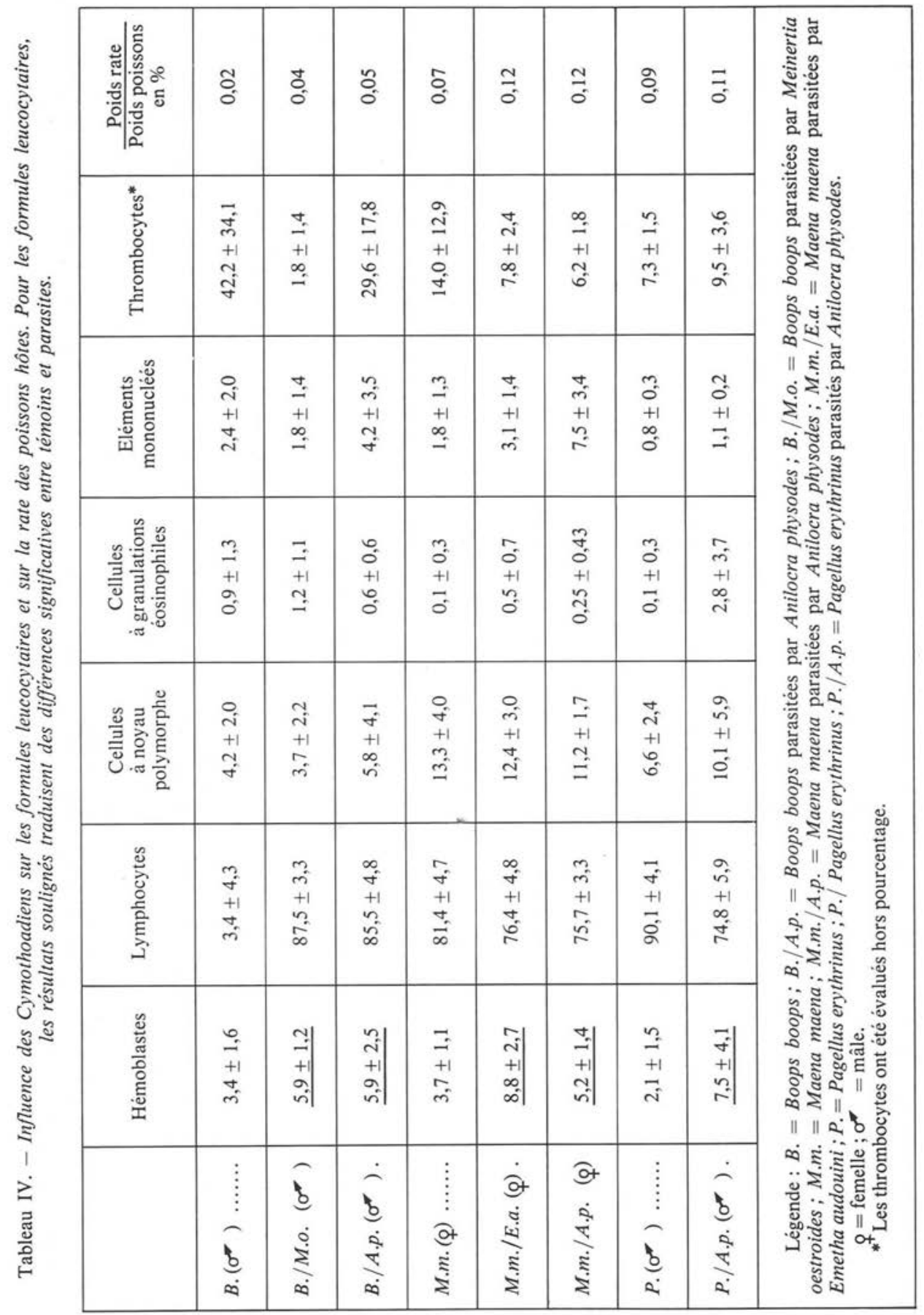


L'interprétation de ces résultats que l'on peut actuellement proposer, est la suivante: la prise de nourriture par les Cymothoadiens est l'équivalent, pour les poissons, d'une suite d'hémorragies. Ces dernières entraînent une anémie des hôtes par ciminution du nombre des érythrocytes circulants. La rate (organe hématopoïétique le plus important), par un mécanisme réflexe, se développe pour produire davantage de globules rouges. Mais un certain nombre d'érythrocytes sont vraisemblablement mis en réserve dans la rate, ce qui augmente sa masse réelle et explique, au moins en partie, son hypertrophie et l'hypervascularisation.

\section{ACTION SUR LA CROISSANCE DES POISSONS.}

Bien que la littérature concernant les influences des Crustacés parasites sur la croissance des poissons soit peu fournie, on peut toutefois y relever un certain nombre de travaux parmi lesquels, certains ont été effectués:

- sur des Copépodes: Mann (1952), Kabata (1958);

- sur des Cymothoidae: Krykhtin (1951) (in Petrushevski et Shulman, 1958) Kroger et Guthrie (1972); Kaczynski et Cannon (1973), Sadzikowski et Wallace (1974), Weinstein et Heck (1977).

Or, ceux de Sadzikowski et Wallace (1974) laissent supposer l'existence possible d'une influence du Cymothoadien Lironeca ovalis (Say) sur la croissance et le développement de Morone americana. Nous avons donc entrepris une étude comparée des relations taille-poids et de la croissance chez les poissons sains et parasités par les Cymothoidae.

- Pour la relation taille-poids, nous avons utilisé une formule dérivée de la relation d'allométrie définie par Teissier (1935): $\mathrm{W}=\mathrm{aLb}$ où $\mathrm{W}$ représente le poids, $\mathrm{L}$ la longueur totale, $\mathrm{a}$ et $\mathrm{b}$ étant des constantes.

\section{Planche IV}

20: Evolution macroscopique de la régression linguale de Boops boops lors des parasitoses à Meinertia oestroides.

1 et 2 : langues de Boops boops non parasitées; 3 à $5:$ première période de la régression ; 6 et $7:$ deuxième phase de la régression.

21: Coupe transversale de l'appendice lingual de Boops boops au niveau de la zone d'implantation du parasite Meinertia oestroides.

22: Une cellule plurinucléée dans le tissu osseux lingual de Boops boops parasitée par Meinertia oestroides.

23: Vues latérales droites du crâne de Maena smaris.

$a=$ individu non parasité $; b=$ individu parasité par Emetha audouini.

24: Apparition de dents vomériennes chez Maema smaris parasitée par Emetha audouini.

25: Coupe transversale de la rate de Boops boops non parasitée.

26: Coupe transversale de la rate de Boops boops parasitée par Anilocra physodes. c.p. = cellules plurinucléées ; c.s. = cellules sanguines ; m.b. = membrane basale ;

d. $=$ derme $;$ d.v. $=$ dents vomériennes $;$ é. $=$ épiderme $; \mathrm{n} .=$ noyau $;$ t.o. $=$ tissu osseux $;$ v.s. $=$ vaisseaux sanguins. 


Pour chaque espèce et chaque catégorie de poissons, des pesées (en grammes) et des mesures de longueur totale (en centimètres) ont été effectuées; des classes de taille de un centimètre ont été établies.
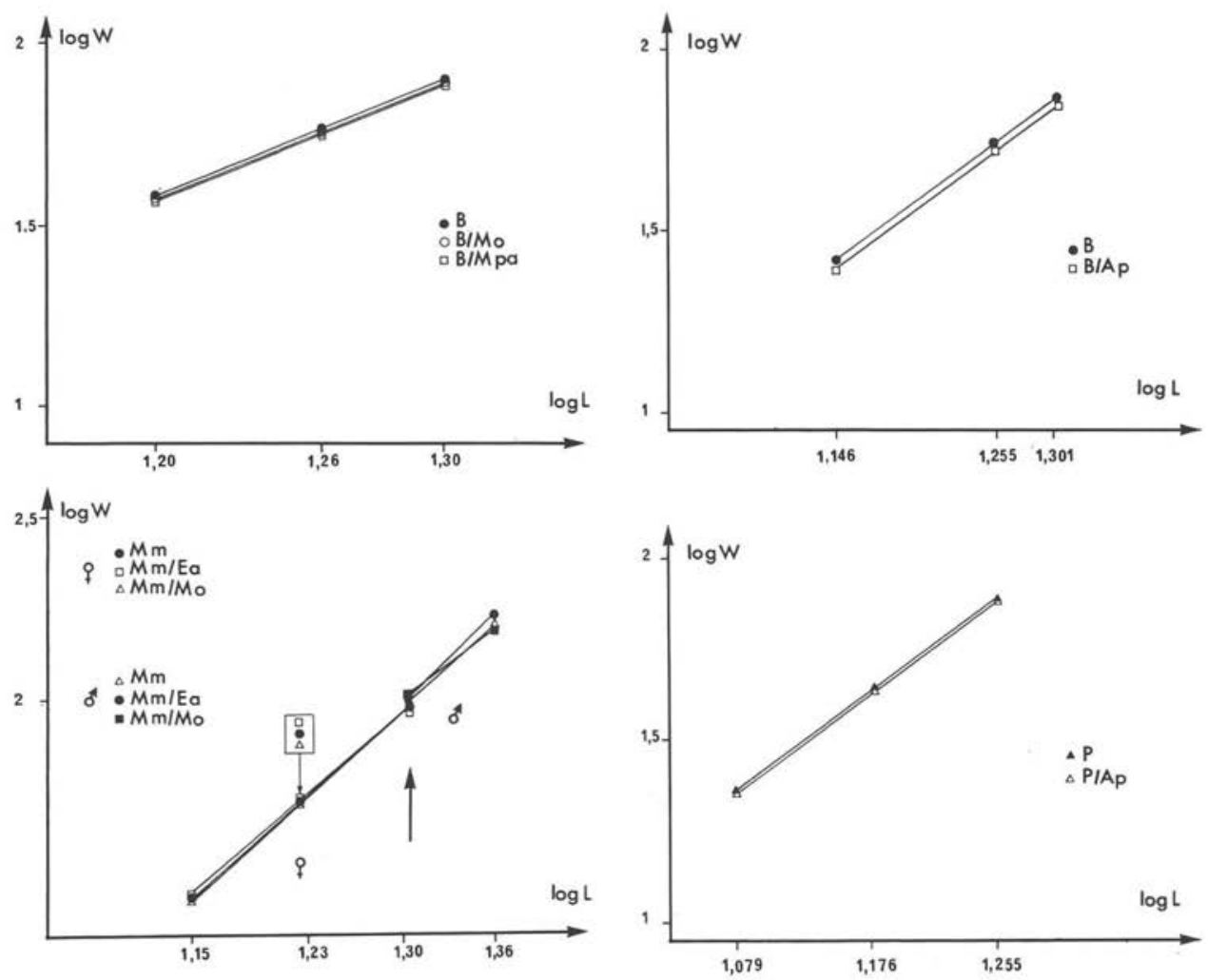

Fig. 1. Les relations taille-poids (coordonnées logarithmiques et droites des moindres rectangles) chez les poissons sains et parasités par les Cymothoidae.

- en abcisse : $\log$ L. = logarithme de la longueur totale.

- en ordonnée: $\log \mathrm{W}=$ logarithme du poids.

$\mathrm{B}=$ Boops boops; $\mathrm{B} / \mathrm{o}=$ Boops boops parasitées par Meinertia oestroides; B/M.pa = Boops boops parasitées par Meinertia parallela; M.m. = Maena maena; Mm/E.a = Maena maena parasitées par Emetha audoui$n i ; \mathrm{Mm} / \mathbf{M} . \mathrm{o}=$ Maena maena parasitées par Meinertia oestroides.

$q=$ femelle $; \delta^{\star}=$ mâle.

Fig. 2. Les relations taille-poids (coordonnées logarithmiques et droites des moindres rectangles) chez les poissons sains et parasités par les Cymothoidae.

— en abcisse; $\log \mathrm{L}=$ logarithme de la longueur totale.

- en ordonnée: $\log \mathrm{W}=$ logarithme du poids.

$\mathrm{B}=$ Boops boops; $\mathrm{B} / \mathrm{A} \cdot \mathrm{p}=$ Boops boops parasitées par Anilocra physodes; $\mathrm{P}=\mathrm{Pa}$ gellus Erythrinus; P/A.p = Pagellus erythrinus parasités par Anilocra physodes. 
Dans tous les cas et pour chaque groupe de taille, les poids moyens des individus infestés sont inférieurs à ceux des témoins. Afin de comparer les différences pondérales observées, nous avons tracé les droites d'ajustement linéaire ou (droites de régression) qui répondent à la formule : $\log \mathrm{W}=\mathrm{b} \log \mathrm{L}+\log \mathrm{a}$; nous avons également calculé les constantes b et a et comparé 2 à 2 à l'intérieur de chaque espèce les valeurs de ces constantes. Ceci nous a permis d'observer qu'il n'existe aucune différence significative à $95 \%$; les valeurs obtenues sont en effet toutes inférieures à 1,96 et les droites correspondantes peuvent être considérées comme confondues (fig. 1 et 2).

- Pour l'étude de la croissance proprement dite, nous avons utilisé la relation $\mathrm{L}=\mathrm{a} \mathrm{T}+\mathrm{b}$ ou $\mathrm{L}$ représente la longueur totale, $\mathrm{T}$ l'âge, $\mathrm{a}$ et $\mathrm{b}$ étant des constantes.

Pour chaque espèce et chaque catégorie de poissons, des mesures de la longueur totale (en centimètres) et des déterminations d'âge (en années), à partir des otolithes, ont été réalisées. Des groupes d'âge de 1 an ont été établis. Dans tous les cas, nous avons pu remarquer qu'à âge égal, les tailles moyennes des poissons parasités sont inférieures à celles des individus sains. Pour comparer ces résultats, nous avons ensuite calculé les constantes a et b (théoriques) des formules $\mathrm{L}=\mathrm{aT}+\mathrm{b}$, tracé ces différentes droites et comparé deux à deux les pentes et les ordonnées à l'origine b. Nous avons ainsi pu noter (fig. 3 et 4 ) :

- aucune différence significative pour les pentes correspondant respectivement à Boops boops saines et parasitées par Meinertia oestroides et aux Maena maena femelles saines et parasitées par Emetha audouini.

- des différences significatives dans le cas des pentes et des ordonnées à l'origine des droites correspondant aux Boops boops saines et parasitées par Meinertia parallela ou par Anilocra physodes et aux Pagellus erythrinus sains et parasités par Anilocra physodes.

Il semble donc que :

- le parasitisme par les Cymothoadiens n'a pas une influence directe, nette sur la relation taille-poids des poissons, bien qu'une légère diminution pondérale soit observée chez les hôtes parasités.

- les Cymothoadiens buccaux et de surface exercent par contre une influence nette, significative, sur la croissance des hôtes. L'influence parasitaire, faible au début de l'infestation, devient progressivement plus importante au cours de la croissance simultanée de l'hôte et de son parasite. Mais l'importance de cette influence paraît dépendre de l'espèce ou de la catégorie écologique des parasites. La comparaison des constantes des droites correspondant aux Boops boops parasitées par Meinertia oestroides et Meinertia parallela par exemple, montre ainsi une action plus importante de la deuxième espèce. 



Fig. 3. Représentations graphiques de la croissance des poissons sains et parasités par les Cymothoidae (valeurs calculées).

- abcisse : $\mathbf{T}=$ âge en ans ;

- ordonnée : $\mathrm{L}=$ Longueur totale en centimètres.

B et B' $^{\prime}=$ Boops boops; $\mathrm{B} / \mathrm{Mo}=$ Boops boops parasitées par Meinertia oestroides; B/M.pa = Boops boops parasitées par Meinertia parallela; M.m = Maena maena; M.m/E.a = Maena maena parasitées par Emetha audouini.

Fig. 4. Représentations graphiques de la croissance des poissons sains et parasités par les Cymothoidae (valeurs calculées).

- abcisse : $\mathbf{T}=$ âge en ans.

- ordonnée : $\mathrm{L}=$ longueur totale en centimètres.

$\mathrm{B}=$ Boops boops; $\mathrm{B} / \mathrm{A} \cdot \mathrm{p}=$ Boops boops parasitées par Anilocra physodes; $\mathrm{P}=\mathrm{Pa}$ gellus erythrinus: P/A.p = Pagellus erythrinus parasités par Anilocra physodes.

\section{Résumé et Conclusions}

Cette étude écophysiologique des parasitoses à Cymothoadiens, nous a donc permis en particulier de mettre en évidence :

- la production par les glandes latéro-œsophagiennes de ces parasites d'une substance antithrombique de nature héparinique active sur le sang des poissons hôtes potentiels des Cymothoidae, des poissons non hôtes potentiels et même sur le 
sang humain. L'hépatopancréas, l'intestin et les diverticules intestinaux postérieurs produisent, quant à eux, des «ferments " hémolytiques actifs sur les érythrocytes du sang des poissons, tandis que l'absorption des sous produits dérivés de l'hémoglobine (hématine et fer) est enfin exclusivement dévolue à l'hépatopancréas.

- l'existence d'une réaction immunologique entre les poissons hôtes (sains et parasités) et les Cymothoadiens ;

- des modifications très spectaculaires des hôtes parasités telles que: la dégénérescence de la langue de Bogues parasitées par Meinertia oestroides; l'apparition de dents vomériennes et les modifications du vomer de Maena smaris parasitées par Emetha audouini;

- des variations biochimiques, hématologiques, histologiques et biométriques chez les poissons, par exemple :

- une diminution de la lipémie et de la teneur en lipides hépatiques,

- une hypomagnésémie,

- une anémie érythrocytaire,

- une augmentation du taux d'hémoblastes circulants,

- une hypertrophie de la rate avec de nombreuses néoformations vasculaires,

- une légère diminution pondérale des hôtes,

- et surtout un retard sur la croissance.

En fait, il ne paraît s'agir là que d'actions relativement modérées et il semble bien exister un certain état d'équilibre, favorable au maintien cuu parasitisme, entre les Cymothoadiens et leurs hôtes.

\section{Bibliographie (*)}

Alexander J. B., Wilson J. G. H., Kersman W. E. (1970) : Immune response in salmon. J. Fish. Biol., 2,384 .

Bowman T.E. (1960): Description and notes on the biology of Livoneca puhi n.sp. (Isopoda, Cymothoidae), parasite of the Hawaïan Moray Eel, Gymnothorax eurostus (Abbott). Crustaceana, I, 84-91.

Brian A. (1912): Di un Isopodo parasita dei pesci (Livoneca sinuata Koelbel). Riv. Mens. Pesca, Idrobiol., VII, 97-99.

Catton W. T. (1951): Blood cell formation in certain teleost fishes. Blood, 6, 39-60.

Christensen J. M. (1964) : Burning of otoliths, a technique for age determination of soles and other fish. J. Cons. Perm. Int. Explor. Mer, 29, 73-81.

De Scalzi G. (1941) : Intorno ad un Isopode Cymothoidae parasita dello Smaris vulgaris. Boll. Pesca Pisci. Idrobiol., XIII, 423-428.

Fain-Maurel M. (1966) : Contribution à l'histologie et à la caryologie de quelques Isopodes : spermiogénèse et infrastructure du spermatozoïde des Oniscidés et des Cymothoidés. Acquisitions récentes sur les spermatogénèses atypiques. Thèse d'Etat, Université Paris, série $\mathrm{A} \mathrm{n}^{\circ} 4468$, $\mathrm{n}^{\circ}$ ordre $5316,1-187$.

(*) Pour une bibliographie plus exhaustive, on pourra consulter notre mémoire original intitulé : « Etude écophysiologique des parasitoses à Cymothoidae. Thèse d'Etat U.S.T.L. Montpellier : 1-284 ; Bibliographie : I à XXXI. 
Folch J., Lees M., Sloane-Stanley G. H. (1957): A simple method for isolation and purification of total lipids from animal tissues. J. Biol. Chem., 226, 497-509.

Gabe M. (1960): Données histochimiques sur les glandes exocrines céphalothoraciques d'Anilocra physodes Leach. Ann. Histochim., 5, 55-63.

Gabe M. (1968): Techniques histologiques. Masson, Paris, 1-1113.

Geitler L. (1953) : Endomitose und endomitotische polyploïdisierung. Protoplasmatologia, 6, C, $1-89$.

Hainline A. J. R. (1958) : Standard methods of clinical chemistry, Academic Press, édit., New York. 2, 1-49.

Harris J.E. (1972): The immune response of cyprinid fish to infections of the acanthocephalan Pomphorhyncus laevis. Int. J. Parasitol., 2, 459-469.

Hodgins H. O., Wieser R. S. Ridgway G. J. (1967): Nature of antibodies and the immune response in rainbow trout. J. Immun., 99, 534-542.

Jakowska S. (1956): Morphologie et romenclature des ceilules da sang des Téléostéens. Rev. Hématol., 11, 519-539.

Johansson-Sjöbeck M. L., Dave G., Larsson A., Lewander K., Lidman U. (1975) : Metabolic and hematological effects of starvation in the European eel: Anguilla anguilla L. II. Hematology. Comp. Biochem. Physiol., 52 A, 431-434.

Johnston M., Davis P. S. (1972) : Carbohydrates of the hepatopancreas and blood tissues of Carcinus. Comp. Biochem. Physiol., 41 B, 433-443.

Kabata Z. (1958) : Lernaeocera obtusa n. s?. Its biology and its effects on the Haddock. Mar. Res., 3. $1-26$

Kaczynski V. W., Cannon T. (1973): Inc.dence and effect of the parasitic Isopod Lironeca ovalis a Bluefish and White Perch in the Lowar Hudson Estuary. Third Symposium on Hudson River Ecology held at Bear Mountain New York.

Kroger R. L.. Guthrie J.-F. (1972): Incidence of the parasitic Isopod Olencira praegustator in juvenile atlantic menhaden. Copeia, 2, 370-374.

Lagarrigue J.G. (1969): Recherches écophysiologiques sur les Oniscoïdes (Isopodes terrestres). Thèse d'Etat, Université Montpellier, n C.N.R.S. AO 3012, 1-258.

Le Cren E. D. (1951): The length-weigth relationship and seasonal cycle in gonad weigth and condition in the perch. J. Anim. Ecol., 20, 201-219.

Legrand J.J. (1952): Contribution à l'étude expérimentale et statistique de la biologie d'Anilocra physodes L. Arch. Zool. Exp. Gen., 89, 1-56

Lellouch J., Lazar P. (1974): Méthodes statistiques en expérimentation biologique. Flammarion, édit. Paris, $\mathrm{n}^{\circ}$ 9171, 1-283.

Lowry O. H., Rosebrough N. H., Farr A. L., Randall R. J. (1951): Protein measurements with the Folin phenol reagent. J. Biol. Chem., 193, 265-275.

Mann H. (1952) : «Lernaeocera branchialis » (Copepoda parasitica) und seine Schadwirkung bei einigen Gadiden. Arch. Fisch. Wiss, 4, 133-144.

Markwardt F., Walsmann P. (1958): Die reaktion zwischen hirudin und thrombin. Hoppe, Seyler's Z. Physiol. Chem., 312, 85-98.

Martin J. (1967): Notions de base en mathématiques et statistiques. Gauthier-Villars, édit., Paris, $1-460$.

Martoja R., Martoja M. (1967) : Initiation aux techniques de l'histologie animale. Masson édit., Paris, 1-345.

Mayrat A. (1959): Nouvelle méthode pour l'étude comparée d'une croissance relative dans deux échantillons. Application à la carapace de Penaeus kerathurus (Forskal). Bull. Inst. Fr. Afr. Noire, 1, 21-59.

Mayrat A. (1967): Croissance et développement chez les Crustacés. Leur étude biométrique (avec quelques remarques sur les insectes). Mem. Inst. Fond. Afr. Notre, A 21, 21-59.

Mayrat A. (1970): Allométrie et taxinomie. Revue statist. appl., 18, 47-58. 
Monod Th. (1976) : Expédition Rumphius II (1975). Crustacés parasites, commensaux, etc.). (Th. Monod et R. Serene, éd.) III - Crustacés Isopodes (1 ${ }^{\text {re }}$ partie) (Corrallanidae, Anilocridae, Cymothoidae). Bull. Mus. Hist. Nat, Paris, 391, 853-870.

Montalenti G. (1949): A new type of polyploïd nucleus in gland cell of Cymothoids (Crustacea, Isopoda) and its cyclic modifications during the phases of activity in cell. Expl. Cell. Res., 1, 123-126.

Montreuil J., Spick G. (1963): Microdosage des glucides totaux. Monographie du Laboratoire de Chimie biologique. Fac. Sci. Lille, 1-145.

Ouchterlony O. (1948): Antigen-antibody reaction in gels. Ark. Kemı. Miner. Geol., $268,1$.

Petrushevski G. K., Shulman S. S. (1958): The parasitic diseases of fishes in the natural waters of the U.S.S.R. in « Parasitology of fishes ». Edt Dogiel (V. A.), Petrushevski (G. K.) et Polyanski (Y. I.). Leningrad University Press, 1958, 1-384.

Poston H. A. (1966) : Effect of sex and reproductive stage on haemoglobin levels in brown trout. Fish. Res. Bull., N. Y., 29, 28-29.

Preston A. (1960): Red blood values in the plaice (Pleuronectes platessa L.). J. Mar. Biol. Ass. U. K., 39, 681-687.

Robertson J. D. (1954): The chemical composition of the blood of some aquatic Chordates, including members of the tunicata, cyclostomata und osteichthyes. J. Exp. Biol., 31, 424-442.

Romestand B., Trilles J.P. (1976) : Au sujet d'une substance à activité antitrombinique mise en évidence dans les glandes latéro-œsophagiennes de Meinertia oestroides (Risso, 1826) (Isopoda, Flabellifera, Cymothoidae ; parasite de poisson). Z. Parasitenk., 50, 87-92.

Romestand B., Trilles J. P. (1977) : Dégénérescence de la langue des Bogues [(Boops boops L., 1758) (Téléostéens, Sparidae)] parasitées par Meinertia oestroides (Risso, 1826) (Isopoda, Flabellifera, Cymothoidae). Z. Parasitenk., 54, 47-53.

Sadzikowski M. R., Wallace D. C. (1974): The incidence of Lironeca ovalis (Say), Crustacea, Isopoda and its effects on the growth of white perch, Morone americana (Gmelin), in the Delaware River near Artificial Island. Chesapeak Sci, 15, 163-164.

Schioedte J. C. (1868): On the structure of the mouth in sucking Crustacea. Ann. Mag. Nat. Hist. Sér. 4, i, 1-25.

Siniscalco M. (1951) : Sulle variazioni morfologicheed istochimiche del nucleo e del citoplasma nelle cellule secretrici di Anilocra physodes. Caryologia, 4, 1-24.

Teissier G. (1935): Les procédés d'étude de la croissance relative. Signification de la loi de dysharmonie. Bull. Soc. Zool. Fr., 60, 292-307.

Trilles J. P. (1968) : Recherches sur les Isopodes Cymothoidae des côtes françaises, $1^{\text {re }}$ thèse : Bionomie, Parasitisme, Biologie générale, Sexualité, 1-793. 2e Thèse: Systématique, Faunistique, 1-181. Thèse d'Etat, Université de Montpellier, $\mathrm{n}^{\circ}$ C.N.R.S. AO 2305.

Von Brand T. (1973) : Biochemistry of parasites. Academic Press edit., New York and London, Second édition, 1-499.

Weinstein M. P., Heck K. L. (1977): Biology and host-parasite relationship of Cymothoa excisa (Isopoda, Cymothoidae) with three species of snappers (Lutjanidae) on the carribbean coast of Panama. Fish. Bull., 75, 875-877.

Yoffey J. M. (1929) : A contribution to the study of the comparative histology and physiology of the spleen with reference chiefly to its cellular constituents. I. in fishes. J. Anat., LXIII, 314-344. 